\title{
Effects of exercise on cancer related fatigue in adults: A literature review and meta-analysis of randomized controlled trials
}

\author{
Suma Vijayakumar*1, Mi-Yeon $\mathrm{Kim}^{2}$, Eric Chan ${ }^{3}$, Ayman Allam ${ }^{4}$ \\ ${ }^{1}$ Department of Nursing, National Center for Cancer Care \& Research, Hamad Medical Corporation, Doha, Qatar \\ ${ }^{2}$ University of Calgary in Qatar, Doha, Qatar \\ ${ }^{3}$ Measurement, Evaluation, and Research Methodology, University of British Columbia, Vancouver, Canada \\ ${ }^{4}$ Department of Palliative care, National Center for Cancer Care \& Research, Hamad Medical Corporation, Doha, Qatar
}

Received: June 19, 2018

DOI: 10.5430/jnep.v9n1p6
Accepted: July 20, 2018

URL: https://doi.org/10.5430/jnep.v9n1p6
Online Published: August 27, 2018

\begin{abstract}
Cancer related fatigue (CRF) is one among the common distressing symptoms experienced by cancer patients. Evidence showed that exercise interventions are effective in decreasing CRF. This review is to evaluate the evidence of the effectiveness of exercise interventions on CRF among adults with varied types of cancer in all phases of the cancer trajectory. A literature review with meta-analysis of randomized controlled trials (RCTs) was conducted. The results of RCTs $(n=20)$ that examined the effects of exercise on CRF were combined using two approaches: meta-analysis $(n=18)$ and summative analysis $(n=2)$. A summary effects size of the standardized mean difference (SMD) with $95 \%$ confidence intervals was calculated using random effects model and heterogeneity was assessed with the $I^{2}$ statistic. The results showed overall, a small but significant decrease in the level of CRF (SMD, $-0.32 ; 95 \%$ CI, -0.51 to $-0.12 ; p=.002$ ) was observed following exercise intervention. Subgroup analyses showed that both mixed modes (combination of resistance and aerobic exercises) and aerobic exercises were effective in significantly reducing CRF ( $p=.033 ; p=.046$ respectively). The results indicated substantial heterogeneity between studies $\left(I^{2}=79 \% ; p \leq\right.$ .0001). Summative analysis also suggested that exercise may be effective in reducing CRF. In conclusion, both resistance and aerobic exercises may be effective in decreasing CRF in adult patients. The result needs to be interpreted with caution due to considerable between-study heterogeneity.
\end{abstract}

Key Words: Cancer fatigue, Exercise, Meta-analysis, Aerobic exercises

\section{INTRODUCTION}

Cancer related fatigue (CRF) is one of the most common, distressing and debilitating symptoms with nearly $91 \%$ of cancer patients in different stages of the disease experience. ${ }^{[1,2]}$ The National Comprehensive Cancer Network (NCCN) defines $\mathrm{CRF}$ as the "distressing, persistent, subjective sense of physical, emotional, and cognitive tiredness or exhaustion related to cancer or cancer treatment, which is not proportional to recent activity, and interferes with usual functioning" (para. 1). ${ }^{[3]} \mathrm{CRF}$ is a multi-dimensional symptom that is different from normal tiredness: it cannot be reversed by rest or sleep ${ }^{[4]}$ and the onset of CRF is more rapid, severe, and energy draining than normal fatigue. ${ }^{[5]}$ The prevalence of CRF varies in different phases of cancer: about $40 \%$ at diagno-

\footnotetext{
*Correspondence: Suma Vijayakumar; Email: svijaykumar434@ gmail.com; Address: Nursing Administration, National Center for Cancer Care \& Research, Hamad Medical Corporation, Doha, Qatar.
} 
sis, ${ }^{[6]} 70 \%$ to $100 \%$ during active cancer treatment, ${ }^{[7]} 30 \%$ during post cancer treatment, ${ }^{[7]}$ more than $75 \%$ in patients with metastatic or advanced stages of cancer, ${ }^{[6]}$ and $17 \%$ to $56 \%$ in long term cancer survivors. ${ }^{[8]}$

Patients with CRF have found their situation debilitating, as it directly interferes with their ability to function physically, mentally, emotionally, spiritually, and socially, resulting in a substantial reduction in their Quality of life (QOL) ${ }^{[9,10]}$ The physical effects of CRF include decreased functional activity leading to a profound inability to perform the day-to-day activities of life. ${ }^{[6]}$ The psychological effects of CRF are feelings of hopelessness, uncertainty, anxiety, mood disturbance, increased depression, loss of employment, and reduced involvement in social activities. ${ }^{[9]}$ Cognitive effects of CRF include an altered thought process, decreased performance of intellectual work, decreased attention span, lowered decision making and problem solving abilities, and difficulty in tackling mental tasks. ${ }^{[4]}$

The pathophysiology of CRF is not well understood. ${ }^{[11]}$ It is presumed that the potential causes and mechanisms of CRF are the tumor itself, the treatment and the accompanying physical and psychological illness. ${ }^{[11]}$ Causes of CRF related to cancer treatment include chemotherapy, radiotherapy, surgery, stem cell transplant, biological response modifiers, and use of concurrent medications such as opioids. ${ }^{[3]}$

There is some evidence that suggests exercise as one of the non-pharmacological interventions to manage the symptoms of CRF. ${ }^{[12]} \mathrm{A}$ variety of exercise interventions have been used for the management of CRF, such as supervised and home-based exercises consisting of low to moderate aerobics, high-intensity resistance training, or a combination of these. ${ }^{[1,10]}$ Exercise is a planned, structured physical activity with the intention to improve or sustain physical or cardiorespiratory fitness. ${ }^{[13]}$ Physical activity is described as any bodily movement generated by skeletal muscle resulting in energy expenditure. ${ }^{[13]}$

The potential benefits of exercise are improved aerobic capacity, physical function, strength, and flexibility to maintain a healthy body composition as well as a healthy body image. ${ }^{[14]}$ Exercise not only impedes and controls cancer, it also improves the physical, mental, and emotional dimensions of fatigue and QOL. ${ }^{[15]}$ Cramp and Byron-Daniel ${ }^{[16]}$ evaluated the effects of exercise on CRF during and after cancer treatment among adult patients with varied types of cancer. They found a statistically significant reduction of fatigue in an exercise group compared to a control group (standardized mean difference [SMD] $-0.27,95 \%$ confidence interval $[\mathrm{CI}]-0.37$ to -0.17$)$. A recent systematic review also supported the benefits of exercise. Paramanandam and Dunn reported that breathing, relaxation, and aerobic exercises are beneficial and safe in managing CRF among lung cancer patients. ${ }^{[15]}$

Different studies have examined the effects of exercise on CRF. But these studies were limited and focused on patients with specific type of tumors such as lung and colorectal cancers, ${ }^{[15,17]}$ and the primary focus was not on fatigue. ${ }^{[17]}$ The only Cochrane review which examined the effect of exercise on CRF in adults was focused on patients with specific cancer trajectory such as during and after cancer treatments. ${ }^{[16]}$ As a result, our understanding of the overall effects of exercise on patients in different cancer trajectory is limited. Therefore, the purpose of this review is to systematically evaluate the current body of evidence on the effectiveness of exercise interventions in influencing the level of CRF among the adult cancer population with varied types of cancer in all phases of the cancer trajectory. The research question framed for this review is "in adults with different types of cancer and in different phases of the cancer trajectory, is exercise intervention more effective in decreasing the level of CRF in comparison to the non-exercise intervention?"

\section{METHOD}

A literature review with a meta-analysis of randomized controlled trials (RCTs) was used to examine the current evidence on the effectiveness of exercise interventions in managing CRF.

\subsection{Search methods}

An initial literature search was conducted in the databases of the Cumulative Index of Nursing and Health Allied Literature (CINHAL), PubMed, MEDLINE, EMBASE, SPORT Discus, Cochrane, and the Middle Eastern and Central Asian Studies. The data search was performed from 15 April 2015 to 15 June 2015 and included research studies from the period between the years 2009 to May 2015. The key search threads used to form the search strategies were exercise interventions, cancer features and fatigue features. The Medical Subject Headings (MeSH) search terms used for the review are detailed in Table 1. The Boolean operator AND was used between these MeSH terms to locate the review topic exercise intervention for the management of CRF in the search strategy.

A three-step search strategy was used for the current review. The limiters year of publication, English language, human, and peer reviewed full text articles were applied to the identified results from each database. In the same sequence, a second search was done using alternate MeSH terms (see Table 1). Finally, a manual search of the key literature, identified by the databases and previous reviews, was carried 
out.

\subsection{Study eligibility criteria}

The format of the population, intervention, comparison, outcome, and study (PICOS) ${ }^{[15]}$ was used to help establish the eligibility criteria for selecting studies to be included in this review. The population $(\mathrm{P})$ included in this review is RCTs that evaluated the effects of exercise on CRF in adults 18 years or older with all types of cancer at varied tumor stages throughout all phases of the cancer trajectory. For the purpose of this review, the cancer trajectory refers to the path inclusive of during, and after any types of cancer treatment, in cancer survivors, and end-stage cancer patients. The cancer survivor in this review refers to anyone who is living with a cancer, after the treatment through the balance of their life. Although, the National Coalition of Cancer Survivorship pioneered an expanded definition of "survivor" as anyone from the time of cancer diagnosis, through the balance of their life. ${ }^{[18]}$ Studies that included patients without a cancer diagnosis or patients concurrent with malignant and non-malignant diseases were excluded.
The intervention (I) included in this review includes those studies in which the treatment group participated in one of two types of interventions: (a) different types of exercises, such as aerobics, resistance, and/or flexibility or (b) a combined mode of aerobics, flexibility, and resistance exercises. The comparison (C) group included in this review includes those studies where the study participants in the comparison groups received usual care or did not receive any treatment. The usual care groups did not receive any specific intervention but received some information, such as how to maintain normal physical activity or a healthy life style. The outcome (O) variable included this review is studies that focused on CRF as the primary or secondary outcome. Further, this review searched for studies that measured CRF using the instruments that were validated. A restriction was not posed on the time of the outcome measure. The study design (S) included in this review is only RCTs of primary studies that were peer reviewed and published in English were considered. The non-experimental studies, or study findings reported in magazines, dissertations and editorials were excluded.

Table 1. Search terms used for the review

\begin{tabular}{ll}
\hline Feature & Search Terms \\
\hline Exercise Intervention & $\begin{array}{l}\text { Exercise, exercise therapy, exercise training, physical activity, aerobic exercise, resistance exercise, } \\
\text { physical training. } \\
\text { Cancer, oncology, malignancy, neoplasm, cancer treatment, chemotherapy, radiotherapy, hormonal } \\
\text { therapy. }\end{array}$ \\
Fatigue Features & Fatigue, cancer related fatigue. \\
\hline
\end{tabular}

\subsection{Study selection process}

Potentially relevant studies were identified from the databases based on the study eligibility criteria. In the initial phase all duplicate studies were removed. In the second phase the title and abstracts were screened for selection against the study eligibility criteria. In the third phase the selected articles were read in full detail to confirm their eligibility. Selected studies were reviewed by two authors to confirm eligibility. Finally, those studies which met the eligibility criteria were selected for the data extraction process.

\subsection{Risk of bias in individual studies}

The Cochrane Collaboration tool was also used to report the risk of bias of individual studies (see Table 2). This tool is comprised of six domains: selection bias, performance bias, detection bias, attrition bias, reporting bias, and other bias. Each domain is rated with three scoring criteria: (a) low risk of bias, which indicates the results are not expected to change significantly; (b) unclear risk of bias, which indicates existence of some doubts about the results; and (c) high risk of bias, which indicates existence of an extreme loss of trust in the results. ${ }^{[38]}$ High risk of bias was considered when there was inadequate information in a particular domain. ${ }^{[38]}$

\subsection{Data extraction}

The data extracted from each of the included studies were author, year of the study, sample characteristics, mode of exercise interventions, outcome variables and measures, and study findings. The extracted data were entered into a predesigned literature review matrix (see Table 3). Data extracted were rechecked by 2 of the authors.

\subsection{Data synthesis and analysis}

The primary outcome measure of this review was the level of CRF in patients with varied malignancies and in different phases of cancer trajectory. To examine the effect of exercise on the level of CRF in cancer patients, the results of 20 RCTs were analyzed and synthesized using two approaches: a meta-analysis and a summative analysis. A meta-analysis was used to statistically combine the results from 18 of the 20 
studies. A summative analysis was used to integrate results from the remaining two studies that were inappropriate to be included in a meta-analysis; in one study, the nature of the intervention was distinctive from the rest of the studies as it was a yoga exercise program, which was specifically designed for breast cancer patients rather than strength based or resistance exercise, and in the second study, the necessary statistics for a meta-analysis were not reported.

Table 2. Assessment of risk of bias using the cochrane collaboration's tool

\begin{tabular}{|c|c|c|c|c|c|c|c|c|}
\hline \multirow[b]{2}{*}{ Studies } & \multicolumn{2}{|c|}{ Selection Bias } & \multirow{2}{*}{$\begin{array}{l}\text { Performance } \\
\text { Bias } \\
\text { Blinding of } \\
\text { Participants } \\
\text { \& Personnel }\end{array}$} & \multirow{2}{*}{$\begin{array}{l}\text { Detection } \\
\text { Bias } \\
\text { Blinding of } \\
\text { Outcome } \\
\text { Assessment }\end{array}$} & \multirow{2}{*}{$\begin{array}{l}\text { Attrition } \\
\text { Bias } \\
\text { Incomplete } \\
\text { Outcome } \\
\text { Data }\end{array}$} & \multirow{2}{*}{$\begin{array}{l}\begin{array}{l}\text { Reporting } \\
\text { Bias }\end{array} \\
\begin{array}{l}\text { Selective } \\
\text { Reporting }\end{array}\end{array}$} & \multirow{2}{*}{$\begin{array}{l}\text { Other Bias } \\
\text { Anything } \\
\text { else, Pre- } \\
\text { specified }\end{array}$} & \multirow{2}{*}{$\begin{array}{l}\text { Total Low } \\
\text { on Risk of } \\
\text { Bias in each } \\
\text { Studies }\end{array}$} \\
\hline & $\begin{array}{l}\text { Random } \\
\text { Sequence } \\
\text { Generation }\end{array}$ & $\begin{array}{l}\text { Allocation } \\
\text { Conceal- } \\
\text { ment }\end{array}$ & & & & & & \\
\hline Adamsen et al. ${ }^{[19]}$ & Low & High & High & High & Low & Low & Low & $4 / 7$ \\
\hline Segal et al. ${ }^{[20]}$ & Low & Low & High & High & Low & low & Low & $5 / 7$ \\
\hline Van Weert et al. ${ }^{[21]}$ & High & Low & High & Low & Low & Low & Low & $5 / 7$ \\
\hline Dodd et. al. ${ }^{[10]}$ & Low* & Low & Low & High & Low & Unclear** & unclear & $4 / 7$ \\
\hline Donnelly et al. ${ }^{[22]}$ & Low & Low & High & High*** & Low & Low & unclear & $4 / 7$ \\
\hline Oldervoll et al. [23] & Low & High & High & High & High & Low & Low & $3 / 7$ \\
\hline Coleman et al. ${ }^{[24]}$ & Low & High & High & High & Low & Low & Low & $4 / 7$ \\
\hline Cho et al. ${ }^{[25]}$ & Low & Low & Low & High & low & Unclear & unclear & $4 / 7$ \\
\hline Yeo et al. ${ }^{[26]}$ & Low & High & High & High & Low & Low & Low & $4 / 7$ \\
\hline Reis et al. ${ }^{[28]}$ & Low & High & High & High & Low & Low & Low & $4 / 7$ \\
\hline Buffart et al. ${ }^{[29]}$ & High & Low & High & Low & Low & Low & Low & $5 / 7$ \\
\hline $\begin{array}{l}\text { Cantarero-Villanueva } \\
\text { et al. }{ }^{[30]}\end{array}$ & Low & Low & Low & Low & Low & Low & Unclear & $6 / 7$ \\
\hline Wenzel et al. ${ }^{[31]}$ & Low & High & High & High & Low & Low & Low & $4 / 7$ \\
\hline Broderick et al. ${ }^{[32]}$ & Low & Low & High & High & Low & Low & Low & $5 / 7$ \\
\hline Andersen et al. [33] & Low & High & High & High & Low & Low & Low & $4 / 7$ \\
\hline Cheville et al. ${ }^{[34]}$ & Low & High & High & High & Low & Low & Low & $4 / 7$ \\
\hline Steindorf et al. ${ }^{[35]}$ & Low & Low & High & High & Low & Low & Low & $5 / 7$ \\
\hline Taso et al. ${ }^{[36]}$ & Low & Low & High & High & Low & Low & Low & $5 / 7$ \\
\hline Cornette et al. ${ }^{[37]}$ & Low & High & High & High & High & Low & Low & $3 / 7$ \\
\hline
\end{tabular}

A statistical procedure was conducted using the Comprehensive Meta-analysis Software, version three. The effect size of the included studies was calculated using Cohen's d, employing a standardized mean difference. The effect sizes were considered statistically significant when a $p$ value of $\leq .05$ was found. When standard deviations (SD) were not reported in the studies, the mean and the $p$ values were used to calculate the Cohen's d effect sizes. When CRF was measured in multiple dimensions, i.e., total fatigue, mental fatigue and physical fatigue, the mean value of the total fatigue was used to calculate the effect size.

The statistical approach of a random-effects model was used because of the anticipated clinical heterogeneity among studies. ${ }^{[39]}$ A random effects model indicates that the effects being assessed in different studies are not identical, but follow some distribution. ${ }^{[39]}$ Heterogeneity among the studies was computed using I-squared $\left(I^{2}\right)$ statistics $^{[40]}$ and Q statistics. ${ }^{[41]}$ The $I^{2}$ statistics represent the approximate proportion of the total variability in point estimates that can lead to heterogeneity. ${ }^{[42]}$ The $\mathrm{Q}$ test evaluates the null hypothesis that there is no variance in the effect sizes across the studies included in the analysis. ${ }^{[43]}$ The $I^{2}$ score of $0 \%$ to $30 \%$, indicates no heterogeneity, $30 \%$ to $49 \%$ moderate heterogeneity, $50 \%$ to $74 \%$ substantial heterogeneity and $75 \%$ to $100 \%$ considerable heterogeneity. ${ }^{[4]}$

\section{RESUlts}

\subsection{Study selection}

Of the 1,122 studies originally identified in the search, 213 remained after the limiters were applied. In addition, a manual search of relevant studies yielded 10 more, resulting in a total of 223 studies. Of the 223 studies, 40 were eliminated due to duplication, and an additional 154 were excluded because they failed to meet the study eligibility criteria. The remaining 29 studies were read in full; subsequently, nine additional studies were excluded because they did not meet the eligibility criteria. Finally, a total of 20 studies were selected to be included in this review (see Figure 1). 
Table 3. Literature review matrix of the 20 studies (randomized controlled trials)

\begin{tabular}{|c|c|c|c|c|c|}
\hline Study & Sample Characteristics & Interventions & Outcome Variables & Outcome Measures & Results \\
\hline $\begin{array}{l}\text { Adamsen } \\
\text { et al. }{ }^{[19]}\end{array}$ & $\begin{array}{l}\mathrm{N}=269, \mathrm{CG}(\mathrm{n})=134, \mathrm{IG}(\mathrm{n})=135 \text {. } \\
\text { Attrition rate }=12.6 \% \text {; Study completion rate } \\
=87.4 \% \text {; Population: Patients with breast, } \\
\text { bowel, and other malignancies; Age } 16-85 \\
\text { years. }\end{array}$ & $\begin{array}{l}\text { IG: Structured high and low } \\
\text { intensity exercise programs: } \\
\text { Cardiovascular and resistance } \\
\text { training, relaxation and body } \\
\text { awareness training. CG: Allowed } \\
\text { to increase physical activity. }\end{array}$ & $\begin{array}{l}\text { Fatigue, } \\
\text { General-wellbeing, } \\
\text { Aerobic capacity, } \\
\text { Muscular strength }\end{array}$ & $\begin{array}{l}\text { Fatigue-European } \\
\text { Organization for } \\
\text { Research and } \\
\text { Treatment of Cancer } \\
\text { Quality of Life (QOL) } \\
\text { Questionnaire } \\
\text { (EORTEC QLQ-30) }\end{array}$ & $\begin{array}{l}\text { Significant improvement in fatigue level } \\
\text { noted in IG at } 6 \text { weeks as compared to } \\
\text { CG. }\end{array}$ \\
\hline $\begin{array}{l}\text { Andersen } \\
\text { et al. }{ }^{[3]}{ }^{2}\end{array}$ & Same as above & Same as above & $\begin{array}{l}\text { Fatigue, General } \\
\text { wellbeing, QOL }\end{array}$ & $\begin{array}{l}\text { Functional } \\
\text { Assessment of Cancer } \\
\text { Therapy-Anemia } \\
\text { Questionnaire; } \\
\text { (FACT-An). }\end{array}$ & $\begin{array}{l}\text { Significant reduction of Fatigue reported } \\
\text { in IG as compared to CG. }\end{array}$ \\
\hline $\begin{array}{l}\text { Van Weert } \\
\text { et al. }[21]\end{array}$ & $\begin{array}{l}\mathrm{N}=209, \mathrm{IG}(\mathrm{n}=147)(\mathrm{PT}=76, \mathrm{PT}+\mathrm{CBT}= \\
71), \mathrm{CG}(\mathrm{n}=62) ; \text { Attrition rate }=5.7 \% \text { Study } \\
\text { completion rate }=94.3 \% \text {; Population: Breast, } \\
\text { hematological, urological, lung, and colon } \\
\text { malignancies; Age } \geq 18 \text { years. }\end{array}$ & $\begin{array}{l}\text { PT group: Aerobic cycle training, } \\
\text { strength training, group sports and } \\
\text { games. All participants: Received } \\
\text { information on exercise } \\
\text { physiology and self-management. }\end{array}$ & Fatigue & $\begin{array}{l}\text { Multi-Dimensional } \\
\text { Fatigue Inventory. }\end{array}$ & $\begin{array}{l}\text { PT group reported significantly lower } \\
\text { level in } 4 \text { domains of fatigue as compared } \\
\text { to CG. General fatigue (GF) }(p=.007), \\
\text { physical fatigue (PF) }(p \leq .001) \text {, mental } \\
\text { fatigue (MF) }(p=.04) \text {. }\end{array}$ \\
\hline $\begin{array}{l}\text { Buffart et } \\
\text { al. }{ }^{[29]}\end{array}$ & Same as above & Same as above & $\begin{array}{l}\text { QOL, Fatigue, } \\
\text { Physical activity, } \\
\text { General efficacy, } \\
\text { Emotional distress. }\end{array}$ & $\begin{array}{l}\text { Multi-dimensional } \\
\text { fatigue inventory. }\end{array}$ & $\begin{array}{l}\text { Had direct effect on fatigue and an } \\
\text { indirect effect through improved physical } \\
\text { activity and general self-efficacy. }\end{array}$ \\
\hline $\begin{array}{l}\text { Segal et } \\
\text { al. }{ }^{20]}\end{array}$ & $\begin{array}{l}\mathrm{N}=121 ; \text { Usual care }(\mathrm{n})=41 ; \text { Resistance } \\
\text { training }(\mathrm{n})=40 ; \text { Aerobic exercise }(\mathrm{n})=40 . \\
\text { Attrition rate }=9 \% \\
\text { Study completion rate }=91 \% ; \text { Population: } \\
\text { Men with prostate cancer. Scheduled to } \\
\text { receive radiotherapy; Mean age }=66.3\end{array}$ & $\begin{array}{l}\text { Resistance training: Exercises } \\
\text { such as leg extensions, leg curls, } \\
\text { and seated chest fly. Aerobic } \\
\text { training: Cycle ergometer, } \\
\text { Treadmill. Usual care: } \\
\text { Maintained usual activity. }\end{array}$ & $\begin{array}{l}\text { Fatigue, QOL, } \\
\text { Physical fitness, } \\
\text { Body composition, } \\
\text { PSA, Testosterone, } \\
\text { Hemoglobin, Lipids. }\end{array}$ & $\begin{array}{l}\text { Functional } \\
\text { Assessment of Cancer } \\
\text { Therapy-Fatigue } \\
\text { (FACT-F Scale) }\end{array}$ & $\begin{array}{l}\text { Fatigue improved with resistance and } \\
\text { aerobic training compared to usual care } \\
\text { from baseline to } 12 \text { weeks. }\end{array}$ \\
\hline $\begin{array}{l}\text { Oldervoll } \\
\text { et al. }{ }^{[23]}\end{array}$ & $\begin{array}{l}\mathrm{N}=231 \text {, IG }(\mathrm{n}=121), \mathrm{CG}(\mathrm{n}=110) \\
\text { Attrition rate }=30 \% \text {; Study completion rate }= \\
70 \% \text {; Population: Metastatic cancer of } \\
\text { gastrointestinal, breast, lung, urological, } \\
\text { hematological, others. Life expectancy } 3 \\
\text { months- } 2 \text { years. PS (Karnofsky) score } \geq 60 . \\
\text { Mean age }=64 \text { years. }\end{array}$ & $\begin{array}{l}\text { Strengthening exercise: Warm up, } \\
\text { circuit training, stretching and } \\
\text { relaxation. Aerobic endurance: } \\
\text { bicycling or treadmill walking. } \\
\text { CG: usual care }\end{array}$ & $\begin{array}{l}\text { Fatigue, Physical } \\
\text { performance }\end{array}$ & $\begin{array}{l}\text { Fatigue Questionnaire } \\
\text { (FQ) }\end{array}$ & $\begin{array}{l}\text { No significant between-groups effects } \\
\text { reported. }\end{array}$ \\
\hline $\begin{array}{l}\text { Coleman et } \\
\text { al. }{ }^{[24]}\end{array}$ & $\begin{array}{l}\mathrm{N}=177, \mathrm{IG}(\mathrm{n}=95), \mathrm{CG}(\mathrm{n}=92) ; \text { Attrition } \\
\text { rate }=11 \% ; \text { Study completion rate }=89 \% \text {. } \\
\text { Population: Newly diagnosed Multiple } \\
\text { Myeloma. Mean age }=56 \text { yrs }\end{array}$ & $\begin{array}{l}\text { IG: Stretching exercise for } \\
\text { hamstrings, shoulder rotation, } \\
\text { calves, and hip flexors, Aerobic } \\
\text { walking. CG: Instructed to remain } \\
\text { active and to walk for } 20 \text { minutes. }\end{array}$ & $\begin{array}{l}\text { Fatigue, Nighttime } \\
\text { sleep, Aerobic } \\
\text { capacity. }\end{array}$ & $\begin{array}{l}\text { Profile of Mood } \\
\text { States (POMS) } \\
\text { fatigue Scale and } \\
\text { FACT-F scale. }\end{array}$ & $\begin{array}{l}\text { No statistical or clinically significant } \\
\text { improvement in fatigue level noted } \\
\text { among the groups. }\end{array}$ \\
\hline $\begin{array}{l}\text { Hayes et } \\
\text { al. }{ }^{277]}\end{array}$ & $\begin{array}{l}\mathrm{N}=194, \text { IG: Face to face }(\mathrm{n}=67)+ \\
\text { Telephone }(\mathrm{n}=67), \mathrm{CG}(\mathrm{n}=60) \text {. Attrition } \\
\text { rate }=7.2 \% ; \text { Study completion rate }=92.8 \% \text {; } \\
\text { Population: Women with breast cancer;Mean } \\
\text { age }=52\end{array}$ & $\begin{array}{l}\text { IG: Aerobics: Mode not reported; } \\
\text { Strength based: Shoulder press } \\
\text { exercise using hand weights. } \\
\text { CG: Received usual care. }\end{array}$ & $\begin{array}{l}\text { QOL, Fitness and } \\
\text { upper body function, } \\
\text { Treatment-related } \\
\text { side effects, (fatigue, } \\
\text { lymphedema, body } \\
\text { mass index, etc.). }\end{array}$ & $\begin{array}{l}\text { Functional } \\
\text { Assessment of } \\
\text { Chronic Illness } \\
\text { Therapy Fatigue } \\
\text { Scale (FACIT-F) }\end{array}$ & $\begin{array}{l}\text { In IG, fatigue improved clinically with } \\
\text { significant fatigue scores in telephone } \\
\text { group. The CG reported with worsening } \\
\text { of fatigue at mid-intervention. }\end{array}$ \\
\hline $\begin{array}{l}\text { Cornette et } \\
\text { al. }{ }^{[37]}\end{array}$ & $\begin{array}{l}\mathrm{N}=44, \mathrm{IG}(\mathrm{n}=22), \mathrm{CG}(\mathrm{n}=22) \\
\text { Attrition rate }=31.8 \% \text {; Study completion rate } \\
=68.2 \% \text {; Population: Women with early stage } \\
\text { breast cancer. Mean age }=50.5 .\end{array}$ & $\begin{array}{l}\text { Aerobic training: cycle ergometer } \\
\text { and walking; Resistance training: } \\
\text { (abdominal hamstrings, } \\
\text { quadriceps, triceps, and gluteus } \\
\text { maximus). CG : Regular activity }\end{array}$ & $\begin{array}{l}\text { Aerobic capacity, } \\
\text { Functional capacity, } \\
\text { Muscle strength, } \\
\text { Fatigue, Anxiety/ } \\
\text { depression, QOL. }\end{array}$ & $\begin{array}{l}\text { Multidimensional } \\
\text { Fatigue Inventory } \\
\text { (MFI-20) }\end{array}$ & $\begin{array}{l}\text { No treatment effect observed between } \\
\text { groups }(p=.157) \text {. }\end{array}$ \\
\hline $\begin{array}{l}\text { Cheville et } \\
\text { al. }+\end{array}$ & $\begin{array}{l}\mathrm{N}=66 ; \mathrm{IG}=33, \mathrm{CG}=33 ; \text { Attrition rate }= \\
15 \% ; \text { Study completion rate }=85 \% \text {; } \\
\text { Population: Stage } 4 \text { Lung and colorectal } \\
\text { cancer, Caucasian ethnicity. Mean age }=64- \\
65.5 \text {. }\end{array}$ & $\begin{array}{l}\text { IG: walking } 1 \mathrm{KM} / 20 \text { minutes or } \\
\text { as much participants can walk } \\
\text { \&Rapid, easy\& strength training } \\
\text { exercise targeting the upper \& } \\
\text { lower body; CG: No instruction } \\
\text { on exercise. }\end{array}$ & $\begin{array}{l}\text { Mobility, Pain \& } \\
\text { sleep quality, } \\
\text { Fatigue, QOL }\end{array}$ & FACT-F & $\begin{array}{l}\text { At } 8 \text { week IG reported improved fatigue } \\
(p=.02) \text {, compared with CG }\end{array}$ \\
\hline $\begin{array}{l}\text { Donnelly et } \\
\text { al. }{ }^{[22]}\end{array}$ & $\begin{array}{l}\mathrm{N}=33 . \mathrm{IG}(\mathrm{n}=16) . \mathrm{CG}(\mathrm{n}=17) \text {. Attrition } \\
\text { rate }=3 \% \text {. Study completion rate }=97 \% \text {. } \\
\text { Population: Women, Gynecological cancer } \\
\text { (stage 1-3). Age } \geq 18 \text {. }\end{array}$ & $\begin{array}{l}\text { IG: Moderate intensity } \\
\text { home-based physical activity } \\
\text { intervention (walking and } \\
\text { strengthening exercise). CG: No } \\
\text { advice }\end{array}$ & Fatigue, QOL & $\begin{array}{l}\text { Multi-dimensional } \\
\text { Fatigue Symptom } \\
\text { Inventory-Short Form } \\
\text { (MFSI-SF), and } \\
\text { FACIT-F }\end{array}$ & $\begin{array}{l}\text { MFSI-SF: Reported significant decrease } \\
\text { in fatigue level among the IG group at } 12 \\
\text { weeks and at } 6 \text { months. FACIT-F: No } \\
\text { statistically significant difference among } \\
\text { the groups was found, but the difference } \\
\text { was clinically significant. }\end{array}$ \\
\hline $\begin{array}{l}\text { Steindorff } \\
\text { et al. }{ }^{.35]}\end{array}$ & $\begin{array}{l}\mathrm{N}=160, \mathrm{IG}(\mathrm{n}=80), \mathrm{CG}(\mathrm{n}=80) ; \text { Attrition } \\
\text { rate }=3 \% ; \text { Study completion rate }=97 \% ; \\
\text { Population: Breast cancer with stage } 0-3, \text { Age } \\
=18 \text { yrs }\end{array}$ & $\begin{array}{l}\text { IG: Received progressive } \\
\text { resistance training. } \\
\text { CG: Received } 12 \text {-week } \\
\text { progressive muscle relaxation }\end{array}$ & Fatigue, QOL & $\begin{array}{l}\text { Fatigue assessment } \\
\text { questionnaire } \\
\text { (FAQ) }\end{array}$ & $\begin{array}{l}\text { IG group }=\text { Significant reduction of total } \\
\text { CRF observed }(p=.044) \text {. CG: No } \\
\text { significant improvement of fatigue noted. }\end{array}$ \\
\hline $\begin{array}{l}\text { Broderick } \\
\text { et al. }{ }^{[32]}\end{array}$ & $\begin{array}{l}\mathrm{N}=43, \mathrm{IG}(\mathrm{n}=23) ; \mathrm{CG}(\mathrm{n}=20) \text {. Attrition } \\
\text { rate }=11.5 \% \text {; Study completion rate }=88.5 \% \text {; } \\
\text { Population: Breast, colon, esophageal, } \\
\text { gynecological cancers and lymphoma. Age }= \\
25-64 \text { years. }\end{array}$ & $\begin{array}{l}\text { IG: Supervised aerobic exercise } \\
\text { (treadmill, rowing machine, and } \\
\text { stationary bicycle); Home-based } \\
\text { exercise (brisk walk). CG: } \\
\text { Normal level of physical activity. }\end{array}$ & $\begin{array}{l}\text { Aerobic fitness } \\
\text { QOL Fatigue } \\
\text { Physical activity }\end{array}$ & FACIT-F & $\begin{array}{l}\text { Improvement in fatigue level (SD, } 6.7 ; p \\
=.01 \text { ) in IG group as compared to CG. }\end{array}$ \\
\hline $\begin{array}{l}\text { Wenzel et } \\
\text { al. }{ }^{[31]}\end{array}$ & $\begin{array}{l}\mathrm{N}=138, \mathrm{IG}(\mathrm{n}=73), \mathrm{CG}(\mathrm{n}=65) \text {. Attrition } \\
\text { rate }=8.6 \% \text {; Study completion rate }=91.4 \% \text {; } \\
\text { Population: male }(61 \%) \& \text { female; All type of } \\
\text { solid tumors (stage1-3). Age } \geq 21 \text {. }\end{array}$ & $\begin{array}{l}\text { IG: Brisk walk. Usual care: } \\
\text { Maintained usual activity. }\end{array}$ & $\begin{array}{l}\text { Fatigue Emotional } \\
\text { distress Sleep } \\
\text { disturbance }\end{array}$ & PFS & $\begin{array}{l}\text { No significant difference between the } \\
\text { exercise and control group noted .More } \\
\text { vigor noted among IG }(p=.03) \text {. }\end{array}$ \\
\hline $\begin{array}{l}\text { Yeo et } \\
\text { al. }{ }^{[26]}\end{array}$ & $\begin{array}{l}\mathrm{N}=110, \mathrm{IG}(\mathrm{n}=54), \mathrm{CG}(\mathrm{n}=48) \text {. } \\
\text { Attrition rate }=13.6 \% \text {; Study completion rate } \\
=86.4 \% \text {; Population: male }(50 \%) \& \text { female } \\
(50 \%) \text { post resection of periampullary cancer; } \\
\text { Mean age }=66-67 .\end{array}$ & $\begin{array}{l}\text { IG: Walking exercise; CG: } \\
\text { Received usual care. }\end{array}$ & $\begin{array}{l}\text { Fatigue Pain } \\
\text { QOL }\end{array}$ & $\begin{array}{l}\text { Visual Analogue } \\
\text { Scale (VAS), } \\
\text { FACIT-F Scale }\end{array}$ & $\begin{array}{l}\text { IG group had significantly improved } \\
\text { fatigue score at the end of the study by } \\
\text { both fatigue scales as compared to CG ( } p \\
=.05 \text { ). }\end{array}$ \\
\hline $\begin{array}{l}\text { Dodd et } \\
\text { al. }{ }^{10]}\end{array}$ & $\begin{array}{l}\mathrm{N}=119, \text { Group }-1(\mathrm{EE}) \mathrm{n}=44 ; \text { Group- } 2(\mathrm{CE}) \\
\mathrm{n}=36 ; \text { Group- } 3(\mathrm{CC}) \mathrm{n}=39 ; \text { Attrition rate }= \\
11 \% \text {; Study completion rate }=89 \% \text {. } \\
\text { Population: Breast, colorectal or ovarian } \\
\text { cancer. Female, } \geq 18 \text { years }\end{array}$ & $\begin{array}{l}\text { Exercise: Moderate intensity } \\
\text { walking, jogging, swimming, and } \\
\text { cycling; EE: Received exercise } \\
\text { prescription and regular follow up } \\
\text { throughout study. CE: Received } \\
\text { exercise prescription and regular } \\
\text { follow-up after the completion of } \\
\text { cancer treatment. CC: Received } \\
\text { only usual care. }\end{array}$ & $\begin{array}{l}\text { Fatigue; Sleep- } \\
\text { disturbance, } \\
\text { Depression, Pain. }\end{array}$ & $\begin{array}{l}\text { Piper Fatigue scale } \\
\text { (PFS) }\end{array}$ & $\begin{array}{l}\text { No significant differences noted between } \\
\text { the groups }\end{array}$ \\
\hline $\begin{array}{l}\text { Cho et } \\
\text { al. }{ }^{[25]}\end{array}$ & Same as above & Same as above & $\begin{array}{l}\text { Duration and } \\
\text { intensity of exercise } \\
\text { dose, Fatigue, Sleep } \\
\text { disturbance, } \\
\text { Depression Pain. }\end{array}$ & $\begin{array}{l}\text { Piper Fatigue Scale } \\
\text { (PFS) }\end{array}$ & $\begin{array}{l}\text { T1 (before chemotherapy): Exercisers } \\
\text { reported significantly lower total fatigue } \\
\text { scores and lower sensory and behavioral } \\
\text { fatigue subscale score. } \\
\text { T2 (End of treatment): Exercisers } \\
\text { reported significantly lower mood/ } \\
\text { cognitive fatigue subscale score than } \\
\text { non-exercisers. } \\
\text { T3 (1 year after T1): No significant } \\
\text { changes noted in fatigue level }\end{array}$ \\
\hline $\begin{array}{l}\text { Cantarero- } \\
\text { Villanueva } \\
\text { et al. }{ }^{[30]}\end{array}$ & $\begin{array}{l}\mathrm{N}=68, \mathrm{IG}(\mathrm{n}=34), \mathrm{CG}(\mathrm{n}=34) \text {. Attrition } \\
\text { rate }=10 \% ; \text { Study completion rate }=90 \% \\
\text { Population: Breast cancer survivors (stage } \\
1-3) \text {. Mean age }=48 \text { years. }\end{array}$ & $\begin{array}{l}\text { IG: Aquatic exercise: aerobic and } \\
\text { endurance exercises; } \\
\text { CG: Information to maintain a } \\
\text { healthy life style. }\end{array}$ & $\begin{array}{l}\text { CRF. Mood state, } \\
\text { Muscular strength } \\
\text { and endurance }\end{array}$ & $\begin{array}{l}\text { Piper Fatigue Scale } \\
\text { (PFS) }\end{array}$ & $\begin{array}{l}\text { IG reported significant reduction of } \\
\text { fatigue in all dimensions and total fatigue } \\
\text { score as compared to CG. }\end{array}$ \\
\hline $\begin{array}{l}\text { Reis } \\
\text { et al. }{ }^{[28]}\end{array}$ & $\begin{array}{l}\mathrm{N}=41 \text {, IG }(\mathrm{n}=22), \mathrm{CG}(\mathrm{n}=19) \text {. Population: } \\
\text { Attrition rate }=\text { Nil; Women with stage I, II, or } \\
\text { III breast cancer, Mean age }=34-85 \text { years. }\end{array}$ & $\begin{array}{l}\text { Nia group: Nia exercises } \\
\text { CG: Usual care }\end{array}$ & $\begin{array}{l}\text { Fatigue, QOL, } \\
\text { Aerobic capacity, } \\
\text { Shoulder flexibility }\end{array}$ & FACIT-F & $\begin{array}{l}\text { The Nia intervention showed } \\
\text { significantly less fatigue as compared to } \\
\text { control group }(p=.05) \text {. }\end{array}$ \\
\hline $\begin{array}{l}\text { Taso et } \\
\text { al. }{ }^{[36]}\end{array}$ & $\begin{array}{l}\mathrm{N}=60 \text {, IG }(\mathrm{n}=30) ; \mathrm{CG}(\mathrm{n}=30) ; \text { Attrition } \\
\text { rate = Nil; Population: Women with stage 1-3 } \\
\text { breast cancer; Age 20-70. }\end{array}$ & $\begin{array}{l}\text { IG: Yoga exercises; CG: } \\
\text { Received usual care. }\end{array}$ & $\begin{array}{l}\text { Anxiety, Depression, } \\
\text { Fatigue }\end{array}$ & $\begin{array}{l}\text { Brief Fatigue } \\
\text { Inventory (BFI) }\end{array}$ & $\begin{array}{l}\text { Reported significant benefits in fatigue } \\
\text { level }\end{array}$ \\
\hline
\end{tabular}




\subsection{Risk of bias within studies}

As shown in Table 2, two studies showed the lowest risk of bias; sixteen studies scored between four and five out of seven, suggesting a low risk of bias; and, two studies showed a high risk of bias. Generally, 18 studies reported a low risk of bias related to random sequence generation, attrition bias and reporting bias. In two studies, random sequence generation was not followed in the control group. ${ }^{[21,29]} \mathrm{Six}$ teen studies reported a high risk of performance bias and detection bias. Detection bias in the studies was likely due to the self-reported nature of the subjective outcome, CRF. Allocation concealment was not reported or not well reported in half of the studies $(n=9)$. Five studies had an unclear risk of other bias that could lead to a high risk of bias because of the infrequent fatigue assessment and self-reported intervention, ${ }^{[10,25]}$ self-reported physical activities, ${ }^{[22]}$ lack of clarity regarding the performance of physical activity during the follow-up period ${ }^{[30]}$ and study limitations that were not reported and were unclear. ${ }^{[27]}$

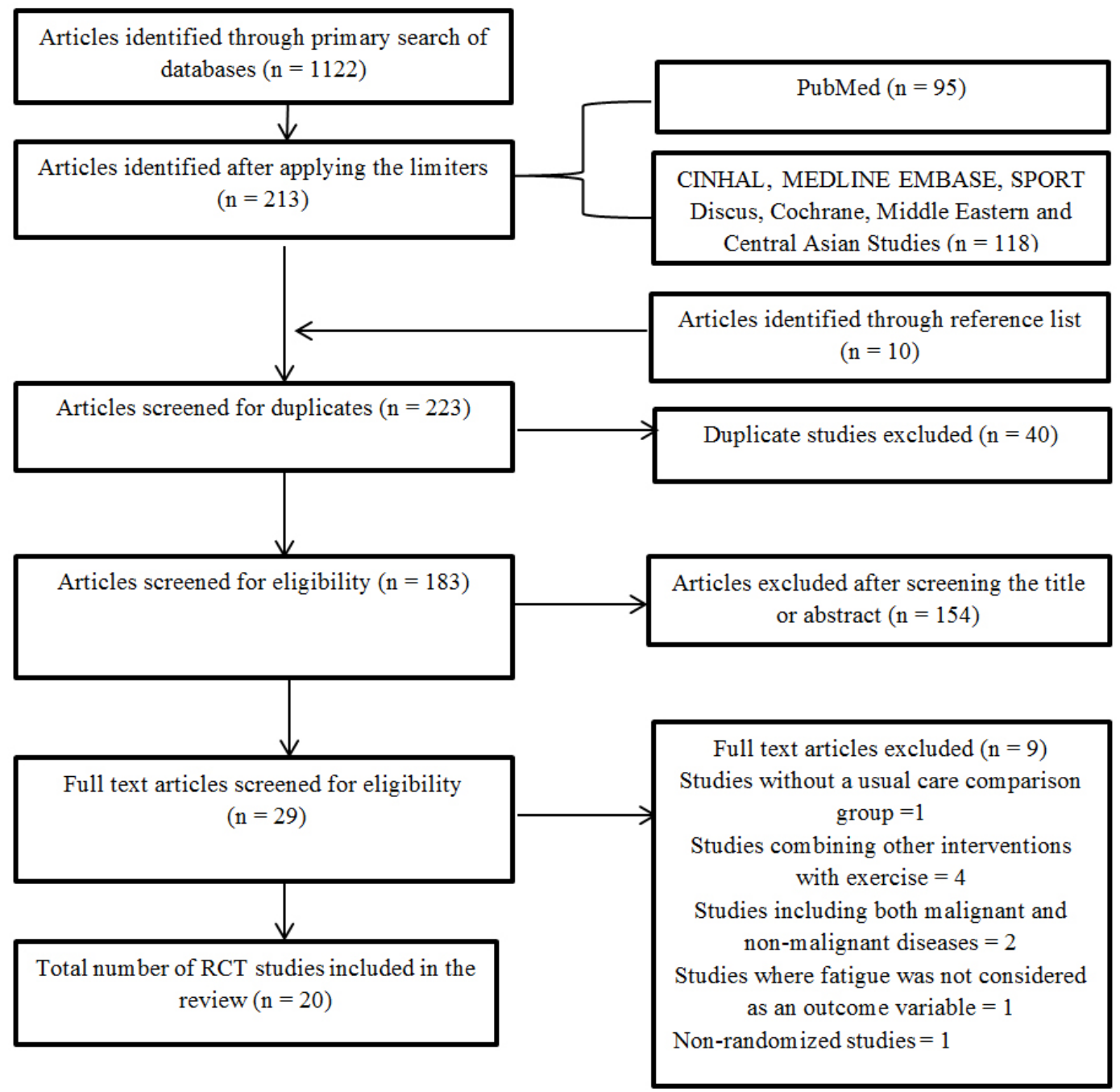

Figure 1. Flow chart of study selection process n: number of articles; RCT: Randomized Controlled Trials

\subsection{Study characteristics}

\subsubsection{Participant characteristics}

A total of 2,083 participants with different types of cancer, varied cancer diagnoses and tumor stages were included in the studies. The range of sample size in each study varied between 33 and 269 participants. Thirteen out of 20 studies

Published by Sciedu Press had a sample size of more than 100, while four studies had a sample size of less than fifty. $71.5 \%$ of study participants were female with the average age of the participants being 55.38 years. The race of the participants was reported in six out of 20 studies were $98 \%$ to $100 \%$ Caucasian.

Participants in the selected studies were diagnosed with var- 
ious types of cancer. Of the 20 studies, 11 included participants who had been diagnosed with the similar type of cancer, such as breast, prostate, multiple myeloma, colorectal, lung malignancies, periampullary adenocarcinoma, or gynecological cancers. The remaining nine studies included participants diagnosed with mixed types of cancer, such as breast, prostate, lung, hematological, colorectal, and other malignancies. Forty-seven percent of the total study participants were diagnosed with breast cancer. Seven other types of cancer affecting the study participants were: gastrointestinal, other malignancies, hematological, prostate, gynecological malignancies, urological, and lung (see Figure 2).

The phases of treatment the participants were receiving in the studies were adjuvant or neo-adjuvant chemotherapy or radiotherapy $(n=11)$, during and after chemotherapy or radiotherapy $(n=3)$, or no treatment which includes end stage diseases $(n=2)$ and the cancer survivors $(n=4)$.

\subsubsection{Modes of exercise intervention}

In this review, 10 out of 20 studies investigated supervised exercise programs or institution-based exercise programs, whereas nine studies investigated home-based or unsupervised exercise programs, and one study used a combined mode of supervised and home based aerobic exercise programs. Four types of exercise were included in the intervention of studies: aerobics, resistance training, a combination of aerobics and resistance training (also referred to as strength-based exercises), and the yoga exercises (see Table $4)$.

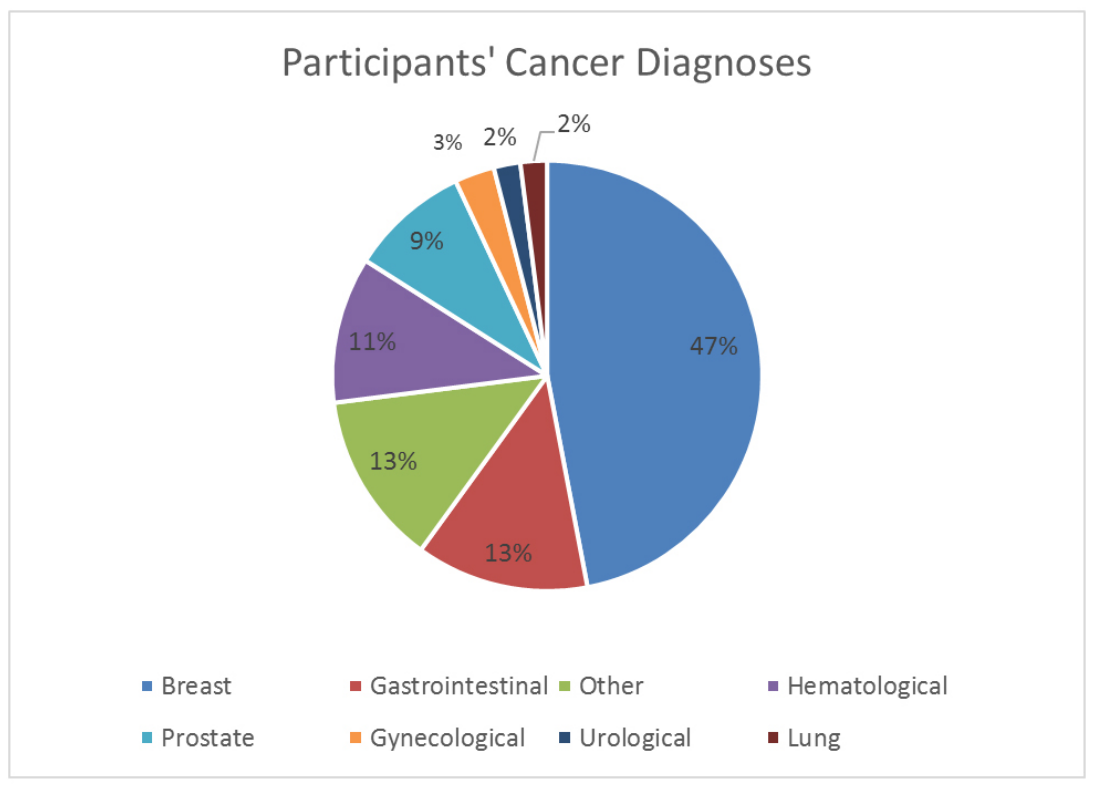

Figure 2. Proportion of study participants' diagnoses by cancer type

The aerobic exercises prescribed in seven of the studies were: a moderate intensity walking program $(\mathrm{n}=2)$; walking along with other modes of aerobic exercises such as cycling, treadmill use, swimming, and/or jogging $(\mathrm{n}=3)$; aquatic aerobics $(\mathrm{n}=1)$ and; a non-traditional Nia exercise $(\mathrm{n}=1)$. The Nia is a cardiovascular and whole-body exercise program that assimilates five aspects of a healthy body: strength, flexibility, mobility, alertness, and stability. ${ }^{[28]}$ Of the different types of aerobic exercise administered, walking was the most commonly prescribed exercise $(n=5)$.

One study prescribed moderate-intensity resistance training exercise for breast cancer patients receiving adjuvant radiation therapy. ${ }^{[35]}$

A mixed mode exercise program which combined aerobics and resistance training (strength based exercises) was prescribed in 11 of the studies. These will be referred to as the mixed modes henceforth. In the mixed mode exercise intervention, the aerobic portion of prescribed exercises consisted of walking, cycling, treadmill use, swimming, and/or jogging. The resistance training portion of prescribed exercises incorporated leg extensions, leg curls, seated chest flies, latissimus pull downs, overhead presses, triceps extensions, biceps curls, calf raises, and/or modified curl-ups. Exercises such as hamstring stretching, shoulder rotations, calf exercises, hip flexor exercises, shoulder presses using hand weights, and climbing up and down steps were incorporated to strengthen upper and lower extremities.

The other type of exercise intervention prescribed was yoga exercise. $^{[36]}$

ISSN 1925-4040 E-ISSN 1925-4059 
Table 4. Modes of exercise intervention used in the studies

\begin{tabular}{|c|c|c|c|c|c|c|}
\hline Studies & Mixed mode & Aerobics & Resistance & Others & Supervised & Un supervised \\
\hline Adamson et al. ${ }^{[19]}$ & $\mathrm{x}$ & & & & $\mathrm{x}$ & \\
\hline Andersen, et al. ${ }^{[33]}$ & $\mathrm{x}$ & & & & $\mathrm{x}$ & \\
\hline Van Weert et al. ${ }^{[21]}$ & $\mathrm{x}$ & & & & $\mathrm{x}$ & \\
\hline Buffart et al. ${ }^{[29]}$ & $\mathrm{x}$ & & & & $\mathrm{x}$ & \\
\hline Segal et al. ${ }^{[20]}$ & $\mathrm{x}$ & & & & $\mathrm{x}$ & \\
\hline Oldervoll et al. ${ }^{[23]}$ & $\mathrm{x}$ & & & & $\mathrm{x}$ & \\
\hline Coleman et al. ${ }^{[24]}$ & $\mathrm{x}$ & & & & & $\mathrm{x}$ \\
\hline Hayes et al. ${ }^{[27]}$ & $\mathrm{x}$ & & & & $\mathrm{x}$ & \\
\hline Cornette et al. ${ }^{[37]}$ & $\mathrm{x}$ & & & & & $\mathrm{x}$ \\
\hline Donnelly et al. ${ }^{[22]}$ & $\mathrm{x}$ & & & & & $\mathrm{x}$ \\
\hline Cheville et al. ${ }^{[34]}$ & $\mathrm{x}$ & & & & & $\mathrm{x}$ \\
\hline Reis et al. ${ }^{[28]}$ & & $\mathrm{x}$ & & & & $\mathrm{x}$ \\
\hline Broderick et al ${ }^{[32]}$ & & $\mathrm{x}$ & & & $\mathrm{x}$ & $\mathrm{x}$ \\
\hline Wenzel et al. ${ }^{[31]}$ & & $\mathrm{x}$ & & & & $\mathrm{x}$ \\
\hline Yeo et al. ${ }^{[26]}$ & & $\mathrm{x}$ & & & & $\mathrm{x}$ \\
\hline Dodd et al. ${ }^{[10]}$ & & $\mathrm{x}$ & & & & $\mathrm{x}$ \\
\hline Cho et al. ${ }^{[25]}$ & & $\mathrm{x}$ & & & & $\mathrm{x}$ \\
\hline Cantarero-Villanueva et al. ${ }^{[30]}$ & & $\mathrm{x}$ & & & $\mathrm{x}$ & \\
\hline Steindorf et al. ${ }^{[35]}$ & & & $\mathrm{x}$ & & $\mathrm{x}$ & \\
\hline Taso et al. ${ }^{[36]}$ & & & & $\mathrm{x}$ & $\mathrm{x}$ & \\
\hline
\end{tabular}

Note. x: studies belong to the modes of exercise intervention.

\subsubsection{Intensity, frequency, and duration of exercise}

The intensity, duration, and frequency of exercise intervention varied among studies. The majority of the studies prescribed moderate intensity aerobics or resistance exercises while Adamson et al., ${ }^{[19]}$ Andersen et al., ${ }^{[33]}$ and Hayes et al. ${ }^{[27]}$ evaluated low-to-high intensity exercises. The duration of exercise varied between 15 to 60 minutes per session. In majority of the studies, the duration of exercise lasted between 120 to 180 minutes per week $(n=13)$. The frequency of exercise ranged from two to five times per week. The length of the exercise intervention varied between six weeks to one year; however, in majority of studies, the exercise intervention lasted for eight to 24 weeks $(n=17)$.

\subsubsection{Outcome measures}

Eleven different instruments were used to measure CRF in the studies included in this review (see Table 5). The most commonly used instruments for the measurement of CRF were the Functional Assessment of Chronic Illness Therapy Fatigue Scale (FACIT-F) and the Piper Fatigue Scale (PFS). Some authors used more than one instrument to measure different dimensions or a degree of clinical significance (in addition to a statistical significance) of fatigue. ${ }^{[22,24,26]}$

Table 5. Fatigue assessment tools used in the review

\begin{tabular}{lc}
\hline Fatigue Tools Used in the Review & $\mathbf{n}$ \\
\hline Functional Assessment of Chronic Illness Therapy Fatigue Scale (FACIT-F) & 6 \\
Piper Fatigue Scale (PFS) & 4 \\
Multidimensional Fatigue Inventory (MFI) & 3 \\
Functional Assessment of Cancer Therapy-Fatigue (FACT-F) & 2 \\
Profile of Mood States (POMS) & 2 \\
Brief Fatigue Inventory (BFI) & 1 \\
Multi-dimensional Fatigue Symptom Inventory-Short Form (MFSI-SF) & 1 \\
Visual Analogue Scale (VAS) & 1 \\
Fatigue Assessment Questionnaire (FAQ) & 2 \\
Functional Assessment of Cancer Therapy-Anemia Questionnaire (FACT-An) & 1 \\
European Organization for Research and Treatment of Cancer Quality of Life Questionnaire (EORTEC-QLQ-30) & 1 \\
\hline
\end{tabular}

Note. n: number of studies. 


\subsubsection{Adverse events}

Except in two studies there were no major adverse events reported during exercise interventions within the reviewed studies. In one study, three participants experienced each of the following episodes: an MI, chest pain and syncope, respectively. ${ }^{[20]}$ In the other study, 25 participants in the intervention group experienced ECG changes during the exercise intervention of walking, swimming, jogging, and cycling-based aerobic exercises. Subsequently, eight of the patients withdrew from the study as they were advised from their primary respective physicians to discontinue with the exercise. $^{[10]}$

\subsection{Meta-analysis}

\subsubsection{Overall effect}

The results of the meta-analysis examining the overall effect of exercise interventions on CRF are presented in Figure 3. The data was pooled using a random effects model, and the standardized mean differences (SMD) with associated confidence intervals (CI) were calculated. The results showed a small but significant effect size that may be indicative of a decreased level of CRF (SMD, -0.32 ; 95\% CI, -0.51 to $-0.12 ; p=.002)$. However, heterogeneity was found to be substantial $\left(\mathrm{Q}=82 ; I^{2}=79 \% ; p=<.0001\right)$, indicating a significant proportion of the overall variation being attributed to between-study variation. The heterogeneity in the effect size distribution could be due to the influence of varied study characteristics or moderator variables, such as variations in diagnoses, exercise interventions and treatment phases. Because of this considerable heterogeneity, sub-group analyses were conducted to explore the possible sources of heterogeneity.

\subsubsection{Sub-group analyses}

The studies were stratified into two sub-groups based on the type of exercise intervention administered in each study. The first sub-group, labelled mixed mode exercises, included participants of studies who received a combination of resistance or strength training and aerobic exercises $(n=10)$. The second sub-group, labelled aerobic exercises, consisted of participants of studies who received exercise intervention of aerobic-type exercises only $(n=7)$. A separate meta-analysis was carried out for each sub-group using a random effects model to account for between-study variations. Random effects model: Test for heterogeneity: $\mathrm{Q}=82$, df $=17, p<$ $.000 \mathrm{I}, I^{2}=79 \%$.

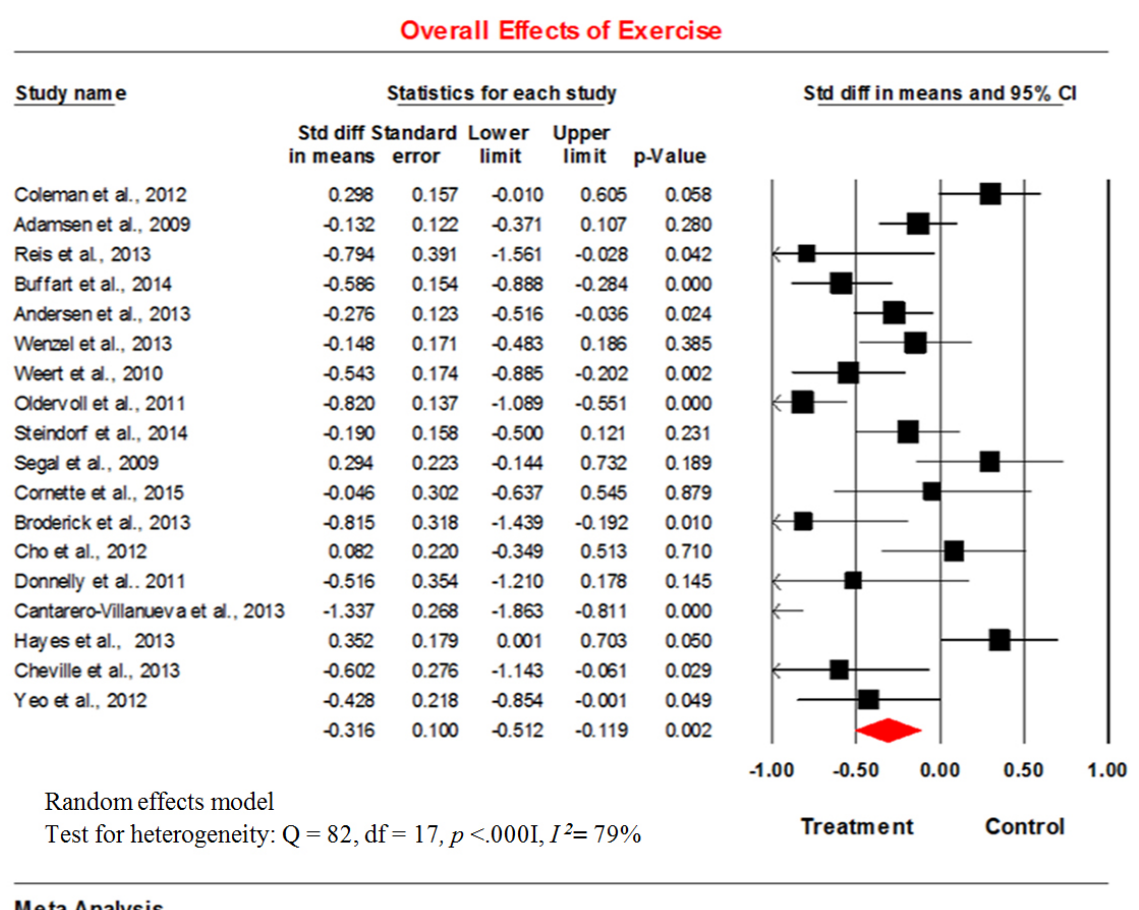

Meta Analysis

Figure 3. Forest plot showing the overall effects of exercise on cancer-related fatigue Note. CI: confidence interval; df: degree of freedom; $I^{2}: I^{2}$ statistics; $Q: Q$ statistics; Std diff: standard difference

The sub-group analysis for the mixed mode exercises group indicated that the exercise intervention may be effective in reducing the level of CRF (see Figure 4). The summary effect size showed a small significant positive change (SMD, $-0.28 ;=52 ; I^{2}=81 \% ; p<.0001$ )
$95 \%$ CI, -0.54 to $-0.02 ; p=.033)$. Similar to the results of the overall effect of the exercise, a considerable heterogeneity was also present in the results for the mixed-modes group $(\mathrm{Q}$

ISSN 1925-4040 E-ISSN 1925-4059 
Mixed Mode Exercises

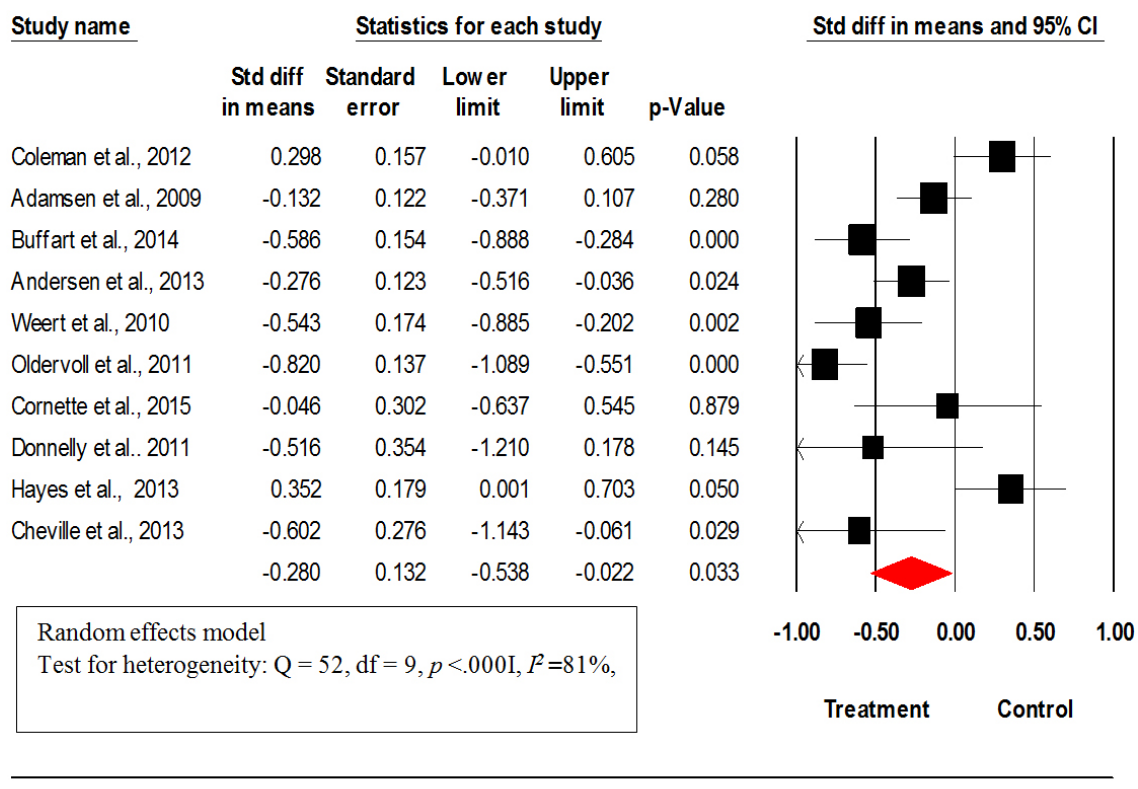

Meta Analysis

Figure 4. Forest plot showing the effects of mixed mode exercise on cancer-related fatigue Note. CI: confidence interval; df: degree of freedom; $I^{2}: I^{2}$ statistics; $Q: Q$ statistics; Std diff: standard difference

The sub-group analysis for the aerobic exercise group showed effect and mixed mode sub-group meta-analysis, the effect a significant moderate summary effect size (SMD, $-0.41 ; 95 \%$ size distribution was found to be considerably heterogeneous CI, -0.82 to $-0.01 ; p=.046)$, indicating that aerobic exer- $\left(\mathrm{Q}=30 ; I^{2}=80 \% ; p \leq .0001\right)$, suggesting the influence of cises may also be effective in decreasing the level of CRF moderator variables in the effect size distribution. (see Figure 5). Again, similar to the results for the overall

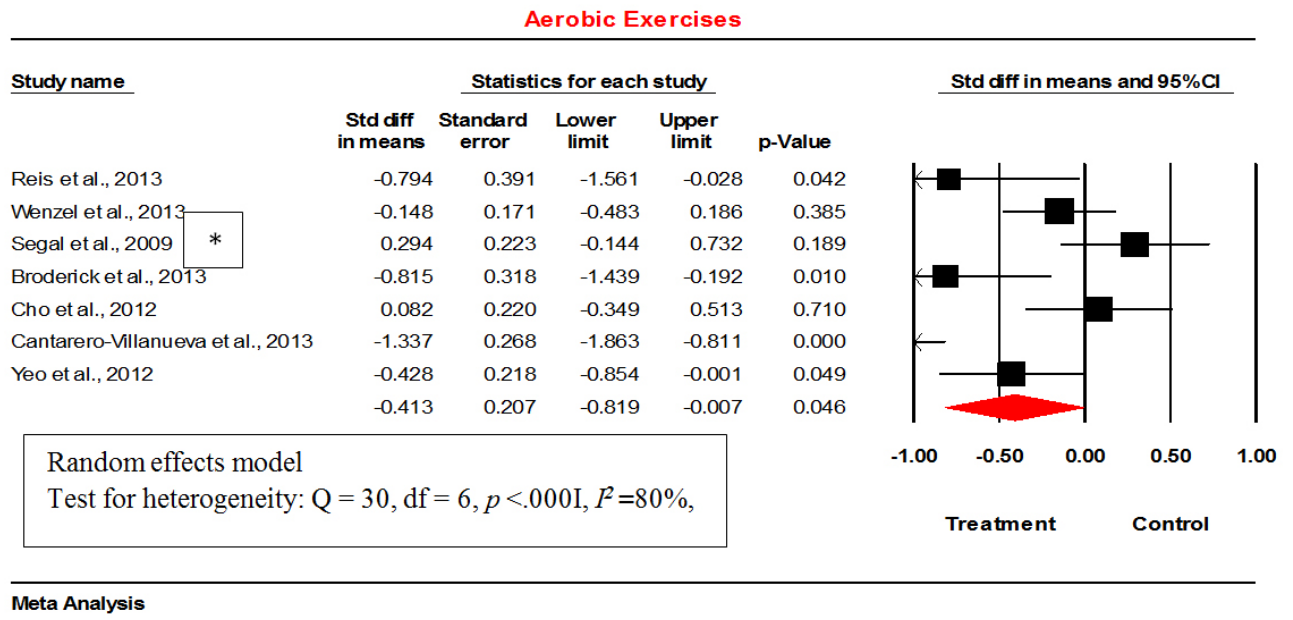

Figure 5. Forest plot showing effects of aerobic exercise on cancer-related fatigue Note. CI: confidence interval; df: degree of freedom; $I^{2}: I^{2}$ statistics; $Q: Q$ statistics; Std diff: standard difference

Although the study by Segal et al. ${ }^{[20]}$ administered both re- tance training are measured as a single outcome, it could not sistance and aerobic exercises together, they measured the be included in the sub-analysis for the mixed-modes group. outcome separately. Since the criterion for mixed-modes Therefore, the aerobic outcome of this study is included in exercises requires that the effects of both aerobics and resis- the aerobic sub-group. 


\subsection{Summative analysis}

Two studies were inappropriate to be included in a metaanalysis as they were analyzed in a summative fashion. Dodd et al.'s ${ }^{[10]}$ study intervention consisting of a home-based moderate intensity aerobic exercise program administered to patients with various types of cancer, such as breast, colorectal, or ovarian, was found to be non-significant in reducing CRF $(p=.84)$. However, a multi-level regression analysis reported significant linear $(p=.02)$ and quadratic $(p=.004)$ effects on fatigue. Finally, Taso et al. ${ }^{[36]}$ administered a supervised yoga exercise intervention to a group of 30 study participants with breast cancer and found that the intervention was significantly effective in managing CRF $(p \leq .001)$.

\section{Discussion}

The key purpose of this review was to examine the evidence on the effectiveness of exercise intervention in reducing the level of CRF among adults with different types of cancer and who are in different phases of the cancer trajectory. This review included 20 RCTs in which the effects of different types, modes, frequencies, and intensities of exercise interventions on cancer patients' CRF were studied. The findings of this review, derived from a meta-analysis of 18 studies ( $p$ $=.002$ ) and summative analyses of one out of two studies, suggest that different types of exercise intervention may significantly decrease the level of CRF in people living with cancer. There is a possibility that exercise interventions may have some potential effect for people with breast, gynecological, and colorectal cancer who are in the early to mid-stages of the cancer trajectory, but further studies are needed. The results of subgroup analyses further demonstrate that both the aerobic $(p=.046)$ and the mixed-modes $(p=.033)$ types of exercise may be significantly effective in decreasing the level of CRF.

Although the results of this review need to be interpreted with caution due to a presence of substantial heterogeneity between studies, the observed positive effect of exercise intervention in managing CRF offers hope that the debilitating effects of fatigue in cancer patients can be managed for better outcomes. Evidence from previous research further supports this optimism in outcome. In the Cochrane review by Cramp and Bryon-Daniel ${ }^{[16]}$ which evaluated the effects of exercise interventions on CRF levels of adult cancer patients during and after cancer treatment, the authors concluded that exercise is a beneficial intervention in moderating the intensity of CRF. Similarly, McMillan and Newhouse ${ }^{[45]}$ who conducted a meta-analysis to examine the effect of exercise intervention on CRF in cancer patients and survivors have also reported that exercise intervention significantly decreased the level of $\mathrm{CRF}(p=.001)$.
The findings of sub-group analyses showed that both the mixed mode and the aerobic-type of exercises significantly reduced the level of CRF in cancer patients. The type of activities included in the mixed mode exercise are walking, cycling and strength based or resistance training, whereas the type of activities included in aerobic exercise are walking and cycling. The benefit of mixed mode exercises found in this review is consistent with the findings of previous research that showed the benefits of decreased CRF from exercises such as aerobics, resistance training and stretching. ${ }^{[45,46]}$ The benefits of aerobic exercise on reducing the level of CRF have been previously reported. ${ }^{[47]}$ The Cochrane review also reported significant benefits of aerobic exercise in reducing CRF among adult patients with varied types of cancer during and after cancer treatment. ${ }^{[16]}$

The findings of the current review seem to suggest that exercises of both the mixed mode and the aerobics are effective intervention for improving CRF in cancer patients, but further studies are needed. However, the conclusion about the effectiveness of all types of exercises may be a little premature as the studies included in this review administered exercises that varied in type, duration, and intensity, and resulted in different outcomes. For example, a short duration, 20-minute, limited intensity, home-based, mixed-modes exercise program was reported to be effective in reducing the level of fatigue in patients with advanced stages of colorectal and lung malignancies $(p=.02){ }^{[34]}$ However, a long-duration, 60-minute, mixed mode of exercise intervention was ineffective in reducing the level of CRF in patients with advanced stages of breast, lung and gastrointestinal malignancies $(p=$ .53). ${ }^{[23]}$ These conflicting outcomes indicate that the longer duration of mixed modes of exercise intervention may not be a realistic option for participants in advanced stages of cancer living with a life expectancy of less than six months. Therefore, more studies are needed to examine the differential impacts of intensity and duration of exercises in participants with advanced stages of cancer.

Although both sub-groups of exercise in this review were significantly effective in decreasing the level of CRF, the magnitude of effect was greater with the aerobic exercise sub-group $(\mathrm{d}=-0.41)$ in comparison to the mixed mode exercise sub-group $(\mathrm{d}=-0.28)$. This suggests that aerobic exercise may be more effective in decreasing the level of CRF in certain circumstances. The differences in the effect size between the aerobic and mixed mode of exercise interventions could be attributed to the characteristics of study participant. Most participants in the aerobic intervention sub-group were females between 40 and 60 years of age with stage 1 to 3 breast cancer. In contrast, participants in the mixed mode sub-group were diagnosed with various types 
of cancer including breast, colorectal and hematological malignancies; some of these participants had advanced stages of cancer with a life expectancy of less than six months. It is plausible that participants in the aerobics sub-group were functionally better and therefore, better able to tolerate the aerobic exercises.

In the two studies not included in the meta-analyses, the participants were female and the majority had malignancies of the breast cancer. ${ }^{[10,36]}$ In these studies, exercise interventions were performed during or after chemotherapy treatment. In one of the studies, the results indicated that the supervised yoga exercise program was significantly effective in improving CRF among women with stage one to three breast cancer undergoing chemotherapy. ${ }^{[36]}$ Similar findings of yoga's beneficial effect on the level of CRF were reported in previous studies involving breast cancer survivors ${ }^{[48]}$ and breast cancer patients undergoing radiation therapy. ${ }^{[49]}$ On the other hand, Dodd et al.' $\mathrm{s}^{[10]}$ home-based moderate intensity walking, jogging and cycling aerobic exercise program showed a nonsignificant effect in reducing CRF among women with breast, colorectal, and ovarian malignancies. This non-significant effect could be related to; infrequent measurement of fatigue level three times in a year is not sufficient to determine the effects of exercise on CRF and/or could be related to the self-reported exercise behavior. However, the exercise group in this study was able to maintain a decreased level of CRF over one year following the intervention in comparison to the control group. ${ }^{[10]}$

The findings of this review also suggest that both supervised and home-based exercise interventions are effective in decreasing CRF. In this review, the home-based exercise interventions incorporated both aerobic and resistance exercises. The beneficial effects of home-based exercises on $\mathrm{CRF}$ and physical functioning have been reported in previous studies. ${ }^{[50,51]}$ Moreover, home-based exercises are feasible, cost-effective, acceptable, and easy to keep up, and hence cancer patients can regularly perform to manage their fatigue levels. ${ }^{[31]}$ Hence, more exercise interventional studies on home-based settings are recommended as the prior studies on home-based exercises are limited due to the trust on supervised exercise programs. ${ }^{[31]}$

The findings of this review suggest that exercise benefits may also be partly attributed to the study participants' type of cancer diagnosis. Although study participants who benefited from the exercise interventions were individuals with breast, prostate, gastro-intestinal, gynecological, and lung cancers; the largest proportion of participants was diagnosed with breast cancer. The positive effects of exercise on CRF in people with breast and prostate cancer have been well

Published by Sciedu Press documented in previous research. ${ }^{[16]}$

In this review, we added new studies and found that exercise may be beneficial in managing CRF. Although exercise appears to be beneficial in managing CRF, further studies are needed to explore the effects of specific type of exercises vs specific types and stages of cancers. In this review, we can't conclude that what type of exercise is beneficial in managing CRF for what type and stages of cancer.

\subsection{Limitations}

A number of limitations arose while conducting this review that need to be considered. First, the considerable heterogeneity observed between studies included in the meta-analyses suggests that the degree of heterogeneity needs to be explored and minimized. For example, the majority of studies were carried out in female population (71\%) and in females with breast cancer (47\%). However, the effects of exercise may differ for males with different types of cancer. In addition, individuals who participated in the exercise interventions differed in the types of cancer they were diagnosed with, e.g., hematological versus solid tumors. Moreover, the type, duration and intensity of exercise intervention administered to study participants also differed. Combining the study findings involving heterogeneous characteristics of participants could have introduced bias to the results. Finally, the findings of this review have limited generalizability because of $98 \%$ $100 \%$ of participants in 6 out 20 studies were Caucasian.

\subsection{Implications for practice}

Findings of this review suggest that all cancer population needs to be screened for CRF, based on the standard practice guidelines such as NCCN, or Pan-Canadian fatigue guidelines. The assessment of CRF should be done using validated fatigue assessment tools. Given the evidence that patients with CRF may benefit from exercises, encourage patients to engage in regular exercise to improve their fatigue level based on their individual performance status and if they are eligible for exercises as recommended by NCCN guidelines. The recommended exercise may be tailored based on individual circumstances and needs such as patients' age, types and stages of cancer, and the physical fitness of patients. ${ }^{[3]}$ Healthcare practitioners involved in providing exercise interventions to cancer patients should also consider strategies that would motivate cancer patients to maintain regular exercises.

\subsection{Implications for future research}

Future research should focus on studies with more homogeneous samples in terms of a specific type and stage of cancer, and the type and intensity of exercise that would allow for a more valid and meaningful comparison of outcomes across 
studies. More home-based exercise interventional studies are needed in evaluating the fatigue level among cancer patients as it is feasible and cost-effective. More studies are also needed to examine the differential impacts of dose by type of exercise on individuals with cancer, especially with end-stage cancer. Quality of life is equally important for all people irrespective of their stage of cancer and yet, people with end-stage cancer are often overlooked for participation in exercise interventional studies. Finally, standardization of CRF measurement in research is vitally important in order to measure the fatigue effectively and to reduce the challenges during the study.

\section{Conclusion}

CRF is an abstract, complex, multidimensional, and subjective symptom which emerges as a consequence of cancer itself, and as a side effect of treatment, resulting in substantial deterioration in a cancer patient's quality of life. Evidence shows that exercise is an effective intervention used in the management of CRF. A variety of exercise interventions have been used in effectively improving CRF, such as aerobics, resistance training, or a mixed mode of exercises in a clinically supervised or home-based setting.

The findings of this review suggest that exercise interventions in general may be beneficial for decreasing the level of CRF in patients with varied cancer diagnoses across different phases of the cancer trajectory. There is not one type of exercise that fits or works for all because different types of exercises are combined even in aerobic or resistant exercises. Therefore, although, exercise appears to be beneficial, further studies are needed to explore whether specific type of exercises may be more beneficial for specific type and stages of cancer.

\section{Conflicts of InTERest Disclosure}

The authors declare that there is no funding or conflicts of interest to disclose.

\section{REFERENCES}

[1] Berger AM, Gerber LH, Mayer DK. Cancer-related fatigue. Cancer. 2012; 118(S8): 2261-2269. PMid:22488700

[2] Reif K, Vries UD, Petermann F, et al. A patient education program is effective in reducing cancer-related fatigue: A multi-centre randomized two-group waiting-list controlled intervention trial. European Journal of Oncology Nursing. 2013; 17(2): 204-213. PMid:22898654 https://doi.org/10.1016/j.ejon.2012.07.002

[3] National Comprehensive Care Network. US ([Inc): Clinical practice guidelines in oncology. [Version 1, 2014; cited March 1, 2015] Available from: http://www.nccn.org/professionals/physi cian_gls/f_guidelines.asp

[4] Given B. Cancer related fatigue: A brief overview of current nursing perspectives and experiences. Clinical Journal of Oncology Nursing $2008 ; 12$.

[5] Wanchai A, Armer JM, Stewart BR. Non pharmacologic supportive strategies to promote quality of life in patients experiencing cancer related fatigue: A systematic review. Clinical Journal of Oncology Nursing. 2011; 15(2): 210-211.

[6] Hofman M, Ryan JL, Figueroa-Moseley CD, et al. Cancer related fatigue: The scale of the problem. The Oncologist. 2007; 12(1): 4 10. PMid:17573451 https://doi.org/10.1634/theoncologis t.12-S1-4

[7] Howell D, Olamn SK, Oliver TK, et al. A pan-Canadian practice guideline and algorithm: Screening, assessment, and supportive care of adults with cancer related fatigue. Current Oncology. 2013; 20 (3): e233-e243.

[8] Lawrence DP, Kupelnick B, Miller K, et al. Evidence report on the occurrence, assessment, and treatment of fatigue in cancer patients. Journal of the National Cancer Institute. Monographs. 2004; (32): 40-50.

[9] Borneman T. Assessment and management of cancer related fatigue. Journal of Hospice \& Palliative Nursing. 2013; 15(2): 77-81. https://doi.org/10.1097/NJH.0b013e318286dc19
[10] Dodd MJ, Cho MH, Miaskowski C, et al. A randomized controlled trial of home-based exercise for cancer-related fatigue in women during and after chemotherapy with or without radiation therapy. Cancer Nursing. 2010; 33(4): 245-257. PMid:20467301 https://doi.org/10.1097/NCC.0b013e3181ddc58c

[11] Wang X. Cancer related fatigue. In Berger AM, Shuster JL, Von Roenn JH. Ed. Principles and practice of palliative care and supportive oncology. 4th ed. Philadelphia, Wolters Kluwer; 2013: 86-87.

[12] Dimeo F, Schwartz S, Wesel N, et al. Effects of an endurance and resistance exercise program on persistent cancer-related fatigue after treatment. Annals of Oncology. 2008.

[13] American College of Sports Medicine. ACSM's guidelines for exercise testing and prescription. Lippincott Williams \& Wilkins; 2013.

[14] Wolin KY, Schwartz AL, Matthews CE, et al. Implementing the exercise guidelines for cancer survivors. The Journal of Supportive Oncology. 2012; 10(5): 171. PMid:22579268 https://doi.org/ $10.1016 / \mathrm{j}$. suponc. 2012.02.001

[15] Paramanandam VS, Dunn V. Exercise for the management of cancerrelated fatigue in lung cancer: A systematic review. European Journal of Cancer Care. 2015; 24(1): 4-14. PMid:24720528 https: //doi.org/10.1111/ecc.12198

[16] Cramp F, Byron-Daniel J. Exercise for the management of cancerrelated fatigue in adults. Cochrane Database of Systematic Reviews. 2012; 11(131): 1-98.

[17] Cramer H, Lauche R, Klose P, et al. A systematic review and metaanalysis of exercise interventions for colorectal cancer patients. European Journal of Cancer Care. 2014; 23(1): 3-14. PMid:23834462 https://doi.org/10.1111/ecc. 12093

[18] National Coalition of Cancer Survivorship. 2017. Available from: ht tps://www . canceradvocacy.org/about-us/our-history/

[19] Adamsen L, Quist M, Andersen C, et al. Effect of a multimodal high intensity exercise intervention in cancer patients undergoing chemotherapy: Randomised controlled trial. BMJ. 2009; 339(b3410): $1-11$. 
[20] Segal RJ, Reid RD, Courneya KS, et al. Randomized controlled trial of resistance or aerobic exercise in men receiving radiation therapy for prostate cancer. Journal of Clinical Oncology. 2009; 27(3): 344351. PMid:19064985 https://doi.org/10.1200/JC0.2007.1 5.4963

[21] van Weert E, May AM, Korstjens I, et al. Cancer-related fatigue and rehabilitation: A randomized controlled multicenter trial comparing physical training combined with cognitive-behavioral therapy with physical training only and with no intervention. Physical Therapy. 2010; 90(10): 1413-1425. PMid:20651011 https: //doi.org/10.2522/ptj.20090212

[22] Donnelly CM, Blaney JM, Lowe-Strong A, et al. A randomized controlled trial testing the feasibility and efficacy of a physical activity behavioral change intervention in managing fatigue with gynecological cancer survivors. Gynecologic Oncology. 2011; 122(3): 618-624. PMid:21689848 https://doi.org/10.1016/j · ygyno. 2011.0 5.029

[23] Oldervoll LM., Loge JH, Lydersen S, et al. Physical exercise for cancer patients with advanced disease: A randomized controlled trial. The Oncologist. 2011; 16(11): 1649-1657. PMid:21948693 https://doi.org/10.1634/theoncologist.2011-0133

[24] Coleman EA, Goodwin JA, Kennedy R, et al. Effects of exercise on fatigue, sleep, and performance: A randomized trial. Oncology Nursing Forum. 2012; 39(5): 468-477. PMid:22940511 https://doi.org/10.1188/12.0NF . 468-477

[25] Cho MH, Dodd MJ, Cooper BA, et al. Comparisons of exercise dose and symptom severity between exercisers and non-exercisers in women during and after cancer treatment. Journal of Pain and Symptom Management. 2012; 43(5): 842-854. PMid:22436836 https://doi.org/10.1016/j.jpainsymman.2011.05.016

[26] Yeo TP, Burrell SA, Sauter PK, et al. A progressive post-resection walking program significantly improves fatigue and health-related quality of life in pancreas and periampullary cancer patients. Journal of the American College of Surgeons. 2012; 214(4): 463475. PMid:22321518 https://doi.org/10.1016/j.jamcolls urg. 2011.12.017

[27] Hayes SC, Rye S, Disipio T, et al. Exercise for health: A randomized, controlled trial evaluating the impact of a pragmatic, translational exercise intervention on the quality of life, function and treatmentrelated side effects following breast cancer. Breast Cancer Research and Treatment. 2013; 137(1): 175-186.

[28] Reis D, Walsh ME, Young-McCaughan S, et al. Effects of Nia exercise in women receiving radiation therapy for breast cancer. Oncology Nursing Forum. 2013; 40(5): E374-E381.

[29] Buffart LM, Ros WJG, Chinapaw MJ, et al. Mediators of physical exercise for improvement in cancer survivors' quality of life. Psycho-Oncology. 2014; 23(3): 330-338. PMid:24123482 https : //doi.org/10.1002/pon.3428

[30] Cantarero-Villanueva I, Fernández-Lao C, Cuesta-Vargas AI, et al. The effectiveness of a deep water aquatic exercise program in cancerrelated fatigue in breast cancer survivors: A randomized controlled trial. Archives of Physical Medicine and Rehabilitation. 2013; 94(2): 221-230.

[31] Wenzel JA, Griffith KA, Shang J, et al. Impact of a home-based walking intervention on outcomes of sleep quality, emotional distress, and fatigue in patients undergoing treatment for solid tumors. The Oncologist. 2013; 18(4): 476-484. PMid:23568000 https://doi.org/10.1634/theoncologist.2012-0278

[32] Broderick JM, Guinan E, Kennedy MJ, et al. Feasibility and efficacy of a supervised exercise intervention in de-conditioned cancer survivors during the early survivorship phase: The PEACH trial. Jour- nal of Cancer Survivorship. 2013; 7(4): 551-562. PMid:23749688 https://doi.org/10.1007/s11764-013-0294-6

[33] Andersen C, Rorth M, Ejlertsen B, et al. The effects of a six-week supervised multimodal exercise intervention during chemotherapy on cancer-related fatigue. European Journal of Oncology Nursing. 2013; 17(3): 331-339. PMid:23084254 https ://doi.org/10.1016/j. ejon.2012.09.003

[34] Cheville AL, Kollasch J, Vandenberg J, et al. A home-based exercise program to improve function, fatigue, and sleep quality in patients with stage IV lung and colorectal cancer: A randomized controlled trial. Journal of Pain and Symptom Management. 2013; 45(5): 811821. PMid:23017624 https://doi.org/10.1016/j.jpainsym man.2012.05.006

[35] Steindorf K, Schmidt ME, Klassen O, et al. Randomized, controlled trial of resistance training in breast cancer patients receiving adjuvant radiotherapy: Results on cancer-related fatigue and quality of life. Annals of Oncology. 2014; 25(11): 2237-2243. PMid:25096607 https://doi.org/10.1093/annonc/mdu374

[36] Taso CJ, Lin HS, Lin WL, et al. The effect of yoga exercise on improving depression, anxiety, and fatigue in women with breast cancer: A randomized controlled trial. Journal of Nursing Research. 2014; 22(3): 155-164. PMid:25111109 https://doi.org/10.1097/jn r.0000000000000044

[37] Cornette T, Vincent F, Mandigout S, et al. Effects of home-based exercise training on vo2 in breast cancer patients under adjuvant or neoadjuvant chemotherapy (sapa). European Journal of Physical and Rehabilitation Medicine. 2015; 52(2): 223-232.

[38] Van Haren IE, Timmerman H, Potting CM, et al. Physical exercise for patients undergoing hematopoietic stem cell transplantation: systematic review and meta-analyses of randomized controlled trials. Physical Therapy. 2013; 93(4): 514-528. PMid:23224217 https://doi.org/10.2522/ptj.20120181

[39] Borenstein M, Hedges L, Rothstein H. Meta-analysis: Fixed effect vs. random effects. Meta-analysis. 2007.

[40] Higgins JP, Thompson SG. Quantifying heterogeneity in a meta-analysis. Statistics in medicine. 2002; 21(11): 1539-1558. PMid:12111919 https://doi.org/10.1002/sim.1186

[41] Cochran, William G. The combination of estimates from different experiments. Biometrics. 1954; 10(1): 101-129. https://doi.or $\mathrm{g} / 10.2307 / 3001666$

[42] O'Connor AM, Sergeant JM, Wang C. Conducting systematic reviews of intervention questions 111: Synthesizing data from intervention studies using meta-analysis. Zoonoses and Public Health. 2014; 61(1): 52-63. PMid:24905996 https://doi.org/10.1111/zph . 12123

[43] Pesut B, Sawatzky R, Stajduhar KI, et al. Educating nurses for palliative care: A scoping review. Journal of Hospice \& Palliative Nursing. 2014; 16(1): 47-54. https://doi.org/10.1097/NJH. 00000000 00000021

[44] Higgins, Julian PT, Green S, et al. Cochrane handbook for systematic reviews of interventions. John Wiley \& Sons; 2011.

[45] McMillan EM, Newhouse IJ. Exercise is an effective treatment modality for reducing cancer-related fatigue and improving physical capacity in cancer patients and survivors: A meta-analysis. Applied Physiology, Nutrition, and Metabolism. 2011; 36(6): 892-903. PMid:22067010 https://doi.org/10.1139/h11-082

[46] Meneses-Echávez JF, González-Jiménez E, Ramírez-Vélez R. Effects of supervised multimodal exercise interventions on cancer-related fatigue: Systematic review and meta-analysis of randomized controlled trials. Bio Med Research International. 2015; 1-13.

[47] Pinto BM, Frierson GM, Rabin C, et al. Home-based physical activity intervention for breast cancer patients. Journal of Clini- 
cal Oncology. 2005; 23(15): 3577-3587. PMid:15908668 https: //doi.org/10.1200/JC0.2005.03.080

[48] Bower JE, Garet D, Sternlieb B, et al. Yoga for persistent fatigue in breast cancer survivors. Cancer. 2012; 118(15): 3766-3775 PMid:22180393 https://doi.org/10.1002/cncr. 26702

[49] Chandwani KD, Perkins G, Nagendra HR, et al. Randomized, controlled trial of yoga in women with breast cancer undergoing radiotherapy. Journal of Clinical Oncology. 2014; 32(10): 1058-1065.
PMid:24590636 https://doi.org/10.1200/JC0.2012.48.27 52

[50] Schwartz AL. Daily fatigue patterns and effect of exercise in women with breast cancer. Cancer Pract. 2000; 8: 16-24. https://doi.or $\mathrm{g} / 10.1046 / \mathrm{j} .1523-5394.2000 .81003 \cdot \mathrm{x}$

[51] Judith K, et al. Effect of exercise on biomarkers, fatigue, sleep disturbances, and depressive symptoms in older women with breast cancer receiving hormonal therapy. Oncology Nursing Forum. 2008; 35(4). 